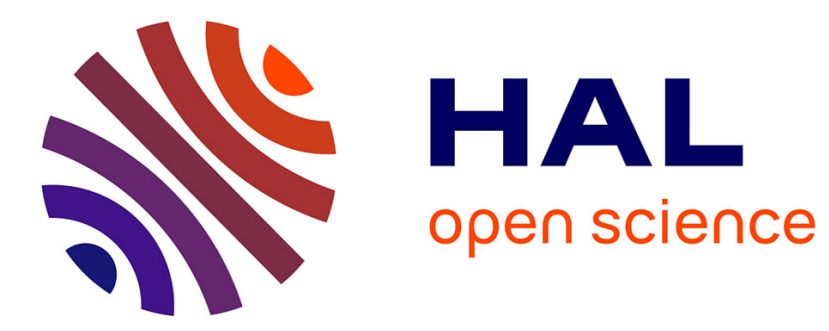

\title{
L'adoption du porcelet, aspects comportementaux et zootechniques
}

E. Deprés, J.C. Caritez

\section{To cite this version:}

E. Deprés, J.C. Caritez. L'adoption du porcelet, aspects comportementaux et zootechniques. Productions Animales, 1991, 4 (2), pp.177-182. hal-00895937

\section{HAL Id: hal-00895937 \\ https://hal.science/hal-00895937}

Submitted on 1 Jan 1991

HAL is a multi-disciplinary open access archive for the deposit and dissemination of scientific research documents, whether they are published or not. The documents may come from teaching and research institutions in France or abroad, or from public or private research centers.
L'archive ouverte pluridisciplinaire HAL, est destinée au dépôt et à la diffusion de documents scientifiques de niveau recherche, publiés ou non, émanant des établissements d'enseignement et de recherche français ou étrangers, des laboratoires publics ou privés. 
INRA Prod. Anim.,

1991, 4 (2), 177 - 182

\section{E. DEPRES, J.C. CARITEZ}

INRA

Domaine Pluridisciplinaire

du Magneraud

BP 52 - 17700 Surgères

\section{L'adoption du porcelet, aspects comportementaux et zootechniques}

\section{En élevage intensif moderne, l'adoption de porcelets est une pratique courante, simple et rapide dans sa mise en place. Toutefois, elle nécessite de la part de l'éleveur un minimum de connaissances et une certaine technicité afin de mieux maîtriser les risques spécifiques. \\ Elle est d'autant plus pratiquée que la conduite en bandes, la synchronisation des mise bas et, dans un avenir proche, le recours à des types génétiques plus prolifiques (lignées Sino-Européennes, lignée hyperprolifique) se généralisent. L'impact économique est relativement important puisqu'il concerne dans certains cas jusqu'à 0,68 porcelet par portée.}

L'amélioration de la productivité numérique du cheptel porcin en France fait l'objet de nombreuses recherches. Les progrès obtenus au cours de la dernière décennie résultent notamment d'une réduction des périodes improductives et de la diminution des pertes de la nais-

\section{Résumé}

L'adoption en élevage intensif moderne est un moyen favorisant la maîtrise de la mortalité des nouveau-nés surnuméraires.

Son importance économique est grande puisqu'elle concerne, dans certaines études, 5,6\% des porcelets nés vivants et 0,61 porcelet sevré par portée. Cette pratique est favorisée par la conduite en bandes, la synchronisation des mise bas et nécessaire avec l'apparition de types génétiques plus prolifiques et la nécessité d'améliorer la productivité numérique de l'élevage. En revanche, ses conséquences sont multiples et affectent particulièrement le comportement du nouveau-né transféré. Cependant, les pertes dues à ces perturbations comportementales sont faibles et l'effet le plus marqué se manifeste surtout sur les performances de croissance du porcelet jusqu'au sevrage et, dans une moindre mesure, jusqu'à l'abattage. Les effets défavorables de cette technique sur les performances de reproduction des truies adoptées sont, quant à eux, limités au taux de conception des nullipares, au nombre de porcelets nés totaux et au nombre de porcelets nés vivants.

L'adoption conduit au nivellement de la taille de la portée en lactation autour d'un optimum variable suivant le type de truie. Elle semble ainsi compenser les effets défavorables d'une taille de portée excessive en lactation.

La période d'intervention, le poids du porcelet transféré, sa vigueur, l'aptitude maternelle de la truie, représentent les principaux facteurs de variation et contribuent ainsi au succès de cette pratique. sance au sevrage (Legault et Molénat, 1982 ; Nogera et al, 1983 ; Ollivier et al 1986).

Un des moyens de diminuer la mortalité post-natale est de transférer d'une portée à l'autre les porcelets en surnombre que la mère ne peut allaiter correctement (production laitière insuffisante, plus de porcelets que de tétines). Cette intervention permet de partager les ressources des truies et contribue ainsi à augmenter les chances de survie des nouveau-nés surnuméraires. Cela revient donc à équilibrer la taille des portées à la naissance autour d'un optimum variable suivant les types génétiques (Deprés et al 1990). Cependant, la taille de portée n'est pas le seul facteur suscitant cette technique : l'interruption accidentelle ou pathologique de la lactation oblige parfois l'éleveur à mettre en place cette pratique.

Cet article présente les principaux résultats bibliographiques concernant l'adoption du porcelet. Dans un premier temps, sont abordées les conséquences de cette pratique sur le comportement, la croissance, la viabilité et les performances de reproduction. La partie suivante est consacrée à l'inventaire des différents facteurs susceptibles de moduler l'efficacité de cette technique.

\section{1 / Adoption et comportement}

Le lien mère-jeune est beaucoup moins fort chez le porc que chez les autres espèces domes- 
tiques (Kilgour 1985) et la truie accepte plus facilement des nouveau-nés étrangers. Lorsque plusieurs femelles sont groupées avec leurs portées, l'adoption de jeunes est fréquemment observée. Par ailleurs, l'apparition du comportement maternel est le résultat d'interactions complexes entre les stimuli externes (visuels, sonores, tactiles et olfactifs) et les mécanismes internes de régulation hormonale au cours de la période périnatale et durant la lactation.

Peu après la naissance, les nouveau-nés établissent des positions régulières au niveau de la mamelle, ils choisissent une tétine qu'ils tendent à conserver par la suite. L'ordre de tétée se développe progressivement dans les jours qui suivent la mise bas : au bout de 3 jours, 50 à $75 \%$ des animaux possèdent une tétine particulière (Hartsock et al 1977). Les interactions agressives sont les plus nombreuses au cours des premières heures et ont lieu surtout au niveau des mamelles antérieures, les plus productives. Le poids à la naissance est un élément déterminant pour la probabilité de succès dans les combats et la possibilité d'accès aux mamelles antérieures. Les animaux dominés sont amenés à changer souvent de tétines (Dantzer et al 1986) et leurs chances de survie sont en général diminuées et leur poids au sevrage inférieur. La liaison mère-jeune interfère de façon très marquée avec la production laitière.

Figure 1. Facteurs comportementaux entrainant une réduction de la quantité de lait ingérée (Horrel et al 1986).

Environnement inconnu pour le porcelet

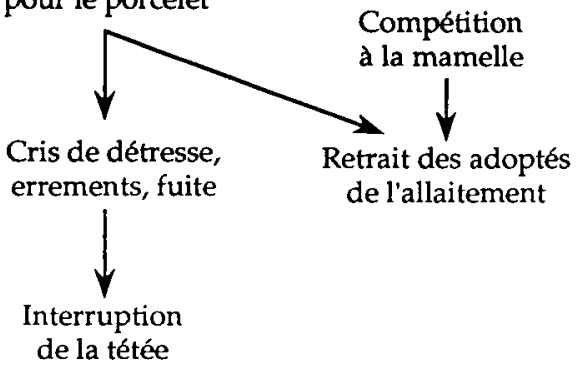

Figure 2. Facteurs comportementaux entrainant une réduction de la quantité de lait produite (Horrel et al 1986).

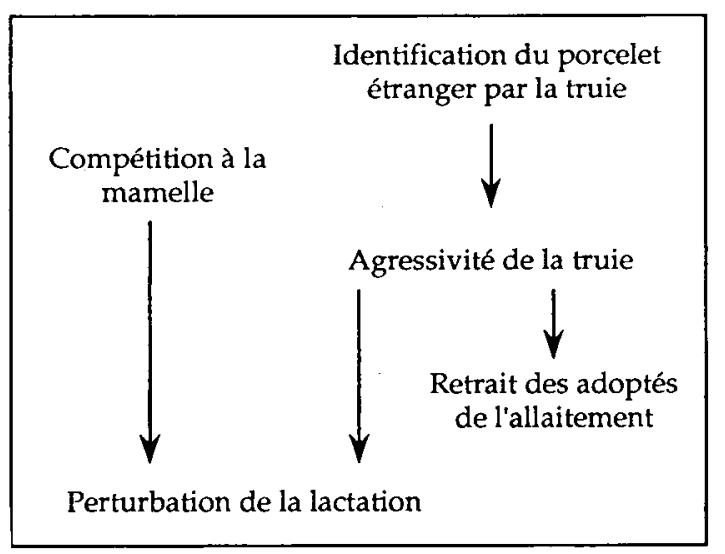

L'adoption est une source importante d'inconfort et de souffrance pour le nouveau-né puisqu'elle désorganise l'établissement des relations sociales entre la truie nourrice et sa portée (Horrel 1982). En effet, on observe, chez les porcelets adoptés, des signes de perturbations du comportement. Ils passent plus de temps à errer en émettant des grognements de détresse caractéristiques et montrent une diminution de l'engagement à la tétée. Dans les heures qui suivent l'adoption, la truie est gênée par l'intensité des combats à la mamelle et les cris que pousse l'étranger au moment de la tétée. D'un point de vue pratique, de tels phénomènes conduisent à la désorganisation de la fréquence et de la durée des tétée (figures 1 et 2). Рar ailleurs, les nouveau-nés transférés qui trouvent au sein des portées nourrices leur tétine «préférée » occupée par un porcelet résident, tendent par la suite à gagner moins de combats.

Les effets de l'adoption sur le comportement du porc peuvent être résumés de la façon suivante (Horrel et Bennet 1981) :

- Désorganisation des relations porcelet-tétine,

- Agressivité de la truie et rejet du porcelet adopté,

- Gêne, inquiétude, excitabilité de la truie nourrice,

- Compétition à la mamelle, agression des porcelets adoptés par les résidents,

- Activités des porcelets adoptés désynchronisées avec celles de la portée d'accueil.

\section{2 / Adoption et viabilité}

L'adoption affecte le comportement à la fois du porcelet transféré, de la truie nourrice et de l'ensemble de la portée adoptive. Aussi, peuton se demander si ce phénomène interfère avec la mortalité post-natale.

Les travaux de Neal et Irvin (1985) montrent que les porcelets non transférés présentent un taux de survie significativement plus élevé $(+9,4 \%)$ comparés aux porcelets adoptés, après ajustement des données selon la vigueur à la naissance. Cependant, ces informations ont été recueillies à partir d'un effectif relativement réduit (254 porcelets issus de 140 portées) et concernent uniquement des truies primipares.

A l'inverse, des résultats récents indiquent que les chances de survie des porcelets transférés ne sont pas affectées par l'adoption. Sur 415 portées nourrices, Deprés et al (1990) observent des taux de survie équivalents chez les porcelets adoptés par rapport à ceux de leurs frères génétiques ou à ceux de leurs frères de lait (figure 3). Ces taux de survie sont, pour cette expérience, élevés et homogènes, les animaux transférés n'occupant pas la plus mauvaise position $(90,8 \%)$. En revanche, les résultats révèlent une variation significative du taux de survie selon le type génétique des truies donneuses et nourrices pour les porcelets résidents. L'analyse précise des causes de mortalité n'ayant pas été réalisée, les auteurs se sont limités à la constatation suivante : sur 109 porcelets adoptés disparus avant 21 jours, $50 \%$ sont morts de faim et de froid suite au stress de l'adoption. 
Figure 3. Survie des porcelets de la naissance à 21 jours (Deprés et al 1990).

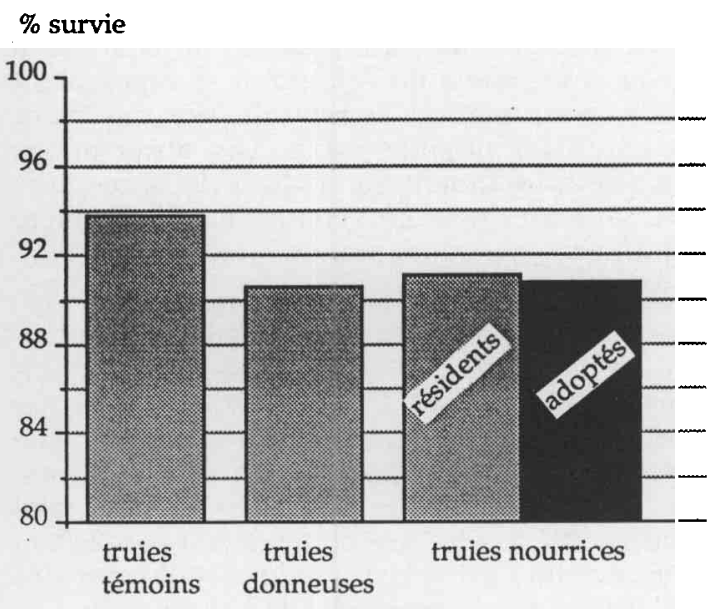

type de portée

Selon Stewart et Diekman (1989), seule la taille de la portée semble influencer la viabilité du porcelet au cours de la lactation. Ces auteurs constatent des taux de survie statistiquement équivalents entre les porcelets adoptés et non adoptés quelles que soient les périodes de la vie de l'animal (tableau 1).

\section{3 / Adoption et croissance}

\section{1 / De la naissance au sevrage}

Le déroulement de l'allaitement est particulièrement affecté par l'adoption de jeunes étrangers. La diminution de l'engagement à la tétée et la perturbation des truies entraînent une réduction de la quantité globale de lait disponible pour le développement du nouveau-né.

Un certain nombre de résultats acquis semblent indiquer que le transfert de porcelets d'une portée à l'autre affecte particulièrement les performances de croissance du jeune au cours de la lactation. Selon la plupart des auteurs, que les porcelets adoptés soient comparés à des témoins, à leurs frères utérins ou leurs frères de lait, leur retard de croissance diffère de manière significative et ce, quels que
Figure 4. Comparaison des estimées des porcelets retirés à leurs "frères utérins" (1) et à leurs "frères de lait " (b) (Deprés et al 1990).
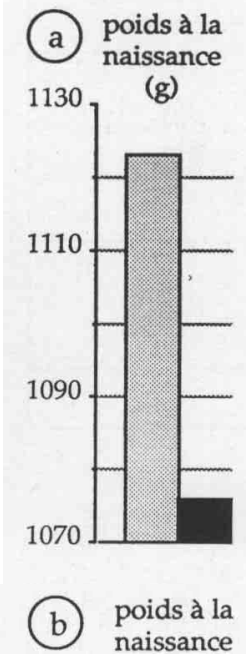

(g)

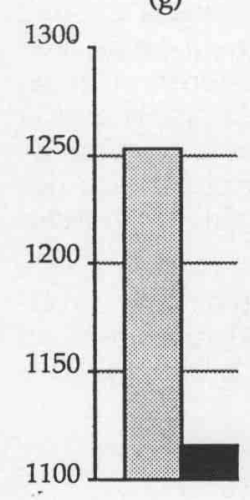

4500

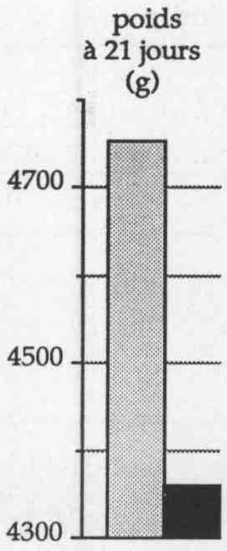

poids

à 21 jours

(g)

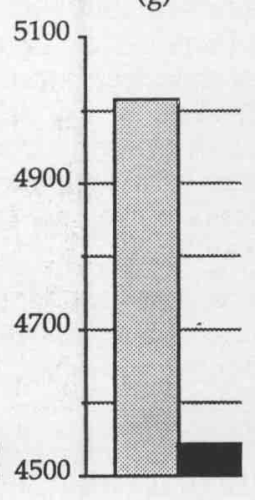

résidents
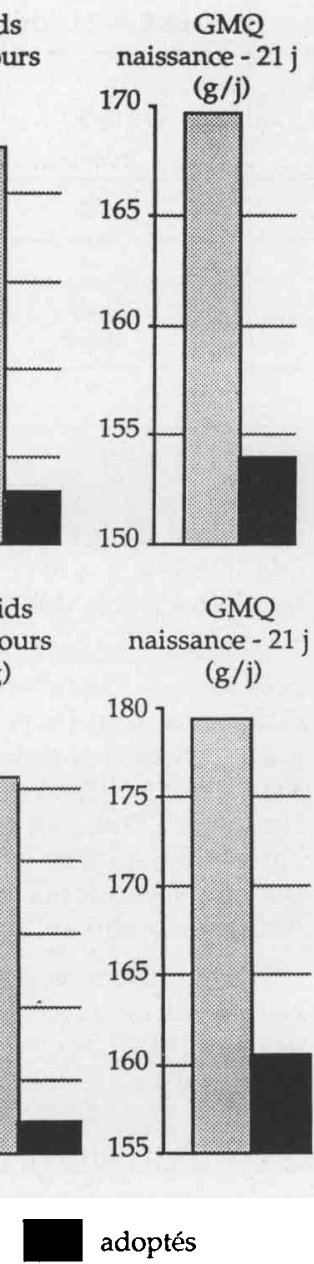

GMQ

naissance $-21 j$

$(\mathrm{g} / \mathrm{j})$

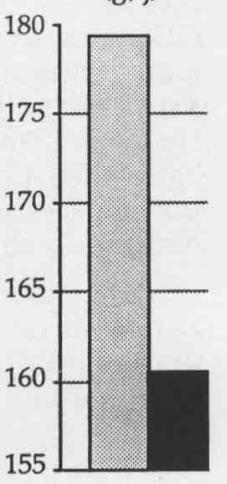

adoptés

soient le type génétique de la mère et l'âge du porcelet au moment de l'adoption.

Les observations réalisées par Deprés et al (1990), mettent en évidence un retard de $16 \mathrm{~g}$ par jour entre les porcelets adoptés et leurs frères utérins ainsi que leurs frères d'allaitement, pour la croissance de la naissance au sevrage (figure 4). Ce handicap de croissance est dû en partie au fait que ce sont les porcelets les moins lourds qui ont été retirés. Dans leur

\begin{tabular}{|l|c|c|c|}
\hline & $\begin{array}{c}\text { Naissance } \\
\text { Sevrage }\end{array}$ & Post-sevrage & $\begin{array}{c}\text { Naissance } \\
\text { Abattage }\end{array}$ \\
\hline $\begin{array}{l}\text { Taille de portée en lactation } \\
\text { 6 porcelets }\end{array}$ & $86 \%$ & $99 \%$ & $85 \%$ \\
$>12$ porcelets & $79 \%$ & $98 \%$ & $77 \%$ \\
\hline & $*$ & NS & NS \\
\hline Adoption & & & \\
Non & $83 \%$ & $98 \%$ & $80 \%$ \\
Oui & $82 \%$ & $98 \%$ & $82 \%$ \\
\hline & NS & NS & NS \\
\hline
\end{tabular}

Tableau 1. Effet de la taille de portée et de l'adoption sur la survie des porcelets (d'après Stewart et Diekman 1989). 
Tableau 2. Effet de la taille de portée et de l'adoption sur la croissance du porc (d'après Stewart et Diekman 1989).

\begin{tabular}{|l|c|c|}
\hline & $\begin{array}{c}\text { Gain en kg } \\
\text { de 0 à 21 jours }\end{array}$ & $\begin{array}{c}\text { Age à 105 kg } \\
\text { en jours }\end{array}$ \\
\hline Taille de portée en lactation & 6,60 & 190 \\
6 porcelets & 5,32 & 195 \\
$>$ 12 porcelets & $* *$ & $*$ \\
\hline Signification & & 191 \\
\hline Adoption & 6,07 & 195 \\
Non & 5,86 & $*$ \\
\hline Oui & $*$ & \\
\hline
\end{tabular}

Différences significatives, ${ }^{*}: \mathrm{P}<0,05 ;{ }^{* *} \mathrm{P}<0,001$.

ensemble, les porcelets adoptés sont plus légers à la naissance que leurs frères utérins $(-47 \mathrm{~g})$ et que leurs frères de lait (-134 g).

D'autres auteurs confirment ces résultats (Stewart et Diekman 1989) et constatent uı différence significative de $210 \mathrm{~g}$ du poids à 21 jours entre les porcelets transférés et leurs frères de lait (tableau 2). Par ailleurs, Horrel et Hodgson (1986), ont montré que la pratique de l'adoption affecte également la croissance des porcelets résidents élevés au sein des portées nourrices (tableau 3).

D'une manière générale le retard de croissance chez les porcelets adoptés est en moyenne inférieur à $500 \mathrm{~g}$ soit moins de $10 \%$ du poids à 21 jours.

\section{2 / Du sevrage à l'abattage}

Les observations recueillies par Neal et Irvin (1985), indiquent que les porcs non transférés ont une croissance plus rapide $(13,5 \%)$ durant la première phase d'engraissement, tandis que les porcs adoptés présentent une croissance compensatrice, non significative, au cours de la période de finition. D'autre part selon Stewart et Diekman (1989) les différences de gain de poids entre les porcelets adoptés et leurs frères génétiques persistent jusqu'à l'abattage. Les nouveau-nés transférés élevés au sein des portées nourrices accusent un retard de 4 jours à $105 \mathrm{~kg}$ (tableau 2).

Pour ce qui concerne l'influence de l'adoption sur les principales variables de la qualité de la carcasse du porc, les résultats indiquent qu'il n'y a pas d'écarts significatifs entre porcelets adoptés et non adoptés (Neal et Irvin 1985).

\section{4 / Adoption et performances de reproduction}

Stewart et Diekman (1989) ont cherché à savoir si les effets de l'adoption se répercutent sur les performances de reproduction des truies au cours du premier cycle. Les observations recueillies indiquent que le taux de conception des nullipares peut être compromis de manière significative par cette pratique (tableau 4).

Par ailleurs les truies élevées au sein des portées adoptives accusent une différence du nombre de porcelets nés totaux et nés vivants, estimée respectivement à 1,5 et 1,7 porcelets par portée (tableau 5). Les auteurs suggèrent que ces femelles sont soumises à un effet défavorable de l'environnement maternel post natal comparable à celui mis en évidence par Nelson et Robinson (1973) et Rutledge (1980), sur des truies nées dans une portée de grande taille.

A l'inverse l'adoption ne semble pas compromettre de manière significative l'âge à la puberté, le pourcentage de cochettes cyclées, le nombre de porcelets sevrés et le poids de la portée à 21 jours ajusté selon les nés vivants.

Stewart et Diekman (1989), mentionnent cependant que, par rapport aux effets défavorables d'une taille de portée en lactation importante, l'impact économique est faible. Avec l'accroissement de la taille de portée en lactation, le taux de survie diminue de 8 points et l'âge à l'abattage augmente de 5 jours alors que les effets négatifs de l'adoption sont quant à eux respectivement estimés à $2-3 \%$ et 4 jours de retard. Ainsi, ils estiment à 0,4 porcelet par portée, la différence entre les truies issues de portées standardisées par l'adoption et les truies élevées au sein des grandes portées. Enfin, ils concluent qu'en limitant les effets maternels défavorables liés à la taille de portée en lactation, les conséquences négatives de l'adoption semblent économiquement compensées. Ils recommandent de ne pas pratiquer l'adoption sur les femelles susceptibles d'être choisies comme reproductrices. Suite à ces observations, il est également conseillé de ne pas choisir les futurs reproducteurs parmi les animaux adoptés.

\section{5 / Facteurs de réussite}

Beaucoup d'initiatives sont laissées à l'éleveur pour pratiquer cette technique, apprécier les capacités laitières et le potentiel maternel de

Tableau 3. Croissance journalière $(g / j)$ des différentes catégories de porcelets et selon la période d'adoption (d'après Horrel et Hodgson 1986).

\begin{tabular}{|l|c|c|c|c|c|}
\hline & \multicolumn{5}{|c|}{ Age en jours } \\
\hline & $\mathbf{1 - 3}$ & $\mathbf{7 - 1 0}$ & $\mathbf{1 4 - 2 0}$ & $\mathbf{2 0 - 3 5}$ & $\mathbf{7 0 - 1 4 0}$ \\
\hline Témoins & 172 & 203 & 209 & 166 & 703 \\
Adoptés 1 jour & 145 & 237 & 235 & 141 & 620 \\
Résidents & 170 & 210 & 205 & 141 & 667 \\
Adoptés 7 jours & 185 & 107 & 185 & 119 & 534 \\
Résidents & 170 & 147 & 208 & 106 & 577 \\
\hline
\end{tabular}


la truie. Toutefois, un certain nombre d'éléments sont susceptibles de moduler l'efficacité de cette pratique.

Comment prévenir et réduire les risques spécifiques liés à cette technique?

D'une manière générale on sait que la meilleure période pour mettre en place cette technique se situe au cours des 24-48 heures postpartum. La liaison mère-jeune est caractérisée par une période privilégiée pour sa mise en place et le fait de pratiquer trop précocement l'adoption compromet l'ingestion de colostrum par le nouveau-né, élément décisif pour la survie de l'animal. Réalisée plus tard, elle entraîne une augmentation de la fréquence des interactions agressives entre porcelet(s) adopté(s) et porcelets résidents (Horrel et Hodgson 1986). La synchronisation des mise bas permet, quant à elle, de transférer des porcelets au sein de portée de même âge et contribue ainsi à équilibrer la taille des portées quelques heures après la naissance.

Un facteur décisif du succès de l'adoption est le poids du porcelet adopté. Deprés et al (1990) indiquent que le taux de survie des porcelets transférés peut être amélioré si on place les nouveau-nés les plus gros au sein des portées les plus âgées et, réciproquement, si on place les porcelets de faible poids au sein des portées les plus jeunes. D'autres facteurs peuvent être à l'origine de l'échec plus ou moins marqué de cette pratique. Neal et Irvin (1985), constatent une corrélation significative entre le taux de survie du porcelet adopté et sa vigueur appréciée à la naissance.

La sélectivité à la tétée se fait essentiellement d'après des critères olfactifs (Meese et Baldwin 1975). Aussi, pour réduire l'agressivité de certaines truies nourrices vis-à-vis des jeunes étrangers, l'éleveur peut masquer l'odeur des porcelets adoptés en mélangeant préalablement ces derniers avec le reste de la portée adoptive.

Enfin, en standardisant la taille des portées à la naissance l'éleveur doit tenir compte du potentiel maternel de la truie car le degré d'adoption varie à l'intérieur d'une même race et surtout, comme l'ont souligné Deprés et al (1990) entre différentes races. En effet, dans cette étude, la proportion des portées donneuses varie de $7 \%$ pour des truies Jiaxing à 32 et $33 \%$ pour des mères Large White et $3 / 4$ Large White. Les proportions de portées nourrices sont également très hétérogènes et varient de 16 à $17 \%$ pour les types prolifiques à $42 \%$ pour les mères Jiaxing. Le nombre de porcelets adoptés ou retirés varie également de manière significative suivant le type génétique de la mère.

\section{Conclusion}

Avec l'apparition de types génétiques plus prolifiques (lignées Sino-Européennes, lignée hyperprolifique) l'adoption devient une obligation au-dessus d'un certain seuil ou plafond 12 à 15 porcelets par portée suivant les types génétiques de truies). La variance phénotypique augmentant aussi, il reste pour l'instant suffisamment de truies moins prolifiques qui constituent un " réservoir de nourrices potentielles ».

Par ailleurs, l'adoption permet le partage des ressources de truies ayant donné des nombres de porcelets différents et conduit alors au nivellement des portées autour d'un optimum variable suivant le type de truie (Deprés et al 1990). Sa réussite dépend de certains facteurs comme le poids du porcelet adopté et sa vigueur et de l'acceptabilité et des qualités maternelles de la truie nourrice.

Tableau 4. Effet de la taille de portée et de l'adoption sur les performances de reproduction des cochettes, d'après Stewart et Diekman 1989.

\begin{tabular}{|l|c|c|c|}
\hline & $\begin{array}{c}\text { Age } \\
\text { à la puberté }\end{array}$ & $\begin{array}{c}\% \text { de cochettes } \\
\text { cyclées }\end{array}$ & $\begin{array}{c}\text { Taux } \\
\text { de conception }\end{array}$ \\
\hline $\begin{array}{l}\text { Taille de portée en lactation } \\
\text { 6 porcelets }\end{array}$ & 225 & 98 & 59 \\
$>12$ porcelets & 229 & 99 & 63 \\
\hline Signification & NS & NS & NS \\
\hline Adoption & & & \\
Non & 226 & 98 & 72 \\
Oui & 228 & 98 & 50 \\
\hline Signification & NS & NS & $*$ \\
\hline
\end{tabular}

Différences significatives, ${ }^{*}: \mathrm{P}<0,05 ;{ }^{* *}: \mathrm{P}<0,001$.

NS : pas de différence significative.

\section{L'adoption \\ des femelles \\ n'entraîne pas \\ de retard \\ à la puberté mais \\ conduit à un taux \\ de conception \\ plus faible \\ au premier cycle.}

\begin{tabular}{|l|c|c|c|}
\hline & $\begin{array}{c}\text { Porcelets } \\
\text { nés totaux }\end{array}$ & $\begin{array}{c}\text { Porcelets } \\
\text { nés vivants }\end{array}$ & $\begin{array}{c}\text { Porcelets } \\
\text { à 21 jours }\end{array}$ \\
\hline $\begin{array}{l}\text { Taille de portée en lactation } \\
\text { 6 porcelets } \\
>\text { 12 porcelets }\end{array}$ & 9,3 & & \\
\hline Signification & 9,0 & 8,2 & $\mathbf{6 , 9}$ \\
\hline $\begin{array}{l}\text { Adoption } \\
\text { Non }\end{array}$ & NS & NS & NS \\
Oui & 9,6 & & \\
\hline Signification & 9,9 & 9,2 & 7,5 \\
\hline
\end{tabular}

Tableau 5. Effet de la taille de portée et de ladoption sur les performances de reproduction des truies en premières portées d'après Stewart et Diekman 1989.

Différences significatives, ${ }^{*}: \mathrm{P}<0,05 ;{ }^{* *}: \mathrm{P}<0,001$.

NS : pas de différence significative. 
English et al (1977) ont montré que, dans certains cas, cette pratique permet de réduire la mortalité post-natale de $40 \%$. Cependant, les expériences rapportées au cours de cette étude montrent que les conséquences de cette pratique sont multiples et affectent le devenir comportemental du jeune. Pourtant les pertes dues à ces perturbations du comportement sont faibles et l'effet le plus grave se manifeste surtout sur les performancës de croissance.

Toutefois, cette technique semble économiquement contrebalancer les effets délétères d'une taille de portée excessive en lactation. On estime généralement que cet effet défavorable équivaut à une réduction de 0,1 porcelet par porcelet supplémentaire dans la portée où la femelle est née (Bidanel 1990).

Pour ce qui concerne le choix des futurs reproducteurs, l'adoption se révèle être un critère à prendre en compte.
En fait, à la lumière des différents travaux présentés dans cet article, il ressort que lorsqu'une source d'inconfort affecte le bien-être du porcelet juste après la naissance, des signes résiduels de perturbation du comportement persistent au-delà de cette période et se répercutent de manière plus ou moins marquée sur les performances de l'animal. L'intervention humaine et notre connaissance incomplète du comportement peuvent alors devenir des facteurs limitants de la productivité de l'élevage.

\section{Remerciements}

Cet article a été réalisé grâce aux conseils de C. Legault, au soutien technique et administratif de C. Bonnet et au travail de l'ensemble de l'équipe INRA «Porcs Chinois » du Domaine du Magneraud. Nous tenons aussi à remercier H. Lagant et Joëlle Lallemand pour leur aide.

\section{Références bibliographiques}

BID NNEL J.P.,1990. Possibilités et perspectives d'amélioration génétique de la prolificité chez le porc. Porc Magazine, 220, pp 63-66.

DANTZER R., AUFFRAY P., SIGNORET J.P., 1986. Le comportement. In : Le porc et son élevage, bases scienti fiques et techniques. Maloine (ed), PARIS, pp 147-149. DEPRES E., CARITEZ J.C., LEGAULT C., LAGANT H. 1990. L'adoption chez le porcelet et ses conséquences. Journées Rech. Porcine en France, 22, 333-338.

ENGLISH P.R., SMITH W.J., MACLEAN A., 1977. The sow - Improving her efficiency, Farming Press Ltd., Ipswich, Suffolk.

HAR'TSOCK TG, GRAVES HB, BAUMGARDI BR, 1977. Agonistic behavior and nursing order in suckling piglets : relationhips with survival growth and body composition. J. Anim. Sci., vol 44, N2, pp 320-330.

HORREL RI, 1982. Immediate behavioural consequences of fostering 1-week-old piglets. J. Agric.Sci., 99, pp 329330.

HORREL I., BENNET J., 1981. Disruption of teat preferences and retardation of growth following cross fostering of 1 -week-old piglets. Anim. Prod., 33, 99-106.

HORREL I., HODGSON T., 1986. The behavioral consequences of fostering in pigs. Ethology of domestics animals. Nichelmann (ed), pp 87-92.

KILGOUR R, 1985. Imprinting in farm animals. In : F, thology of farm animals, Elsevier (ed) chapter 12, pp 133-145.
LEGAULT C., MOLENAT M., 1982. Exposé ENSAM Montpellier Polycopié 13.

MEESE G.B., BALDWIN B.A., 1975. Effects of olfactory bulb ablation on maternal behaviour in sows. Appl. Anim. Ethol., 1, 379-386.

NEAL S.M., IRVIN K.M., 1985. The effect of transferring pigs on survival growth and carcass composition. J. Anim. Sci., 61 (suppl.1), 74.

NELSON R.E., ROBINSON O.W., 1973. Effects of maternal postnatal environnemnt on reproduction of gilts. J. Anim. Sci., 43-71.

NOGERA JL., FELGINES C., LEGAULT C, 1983 , Fvolution de 1972 à 1981 des composantes de la productivité numérique des truies dans 325 troupeaux Français. Journées de la Rech. Porcine en France, 15, 37-42.

OLLIVIER L., RUNAVOT JP., DAGORN J., GUEBLEZ R., JEHANNO J., KERESIT R., LEGAULT C., MOLENAT M., SELLIER P., 1986. La loi sur l'élevage de 1966; un bilan de 20 années de sélection rationnelle du porc en France. Journées de la recherche Porcine en France, 18, 203-236.

RUTLFDGE J.J., 1980. Fraternity size and swine reproduction. Effect of fecundity of gilts. J. Anim. Sci. 51-868.

STEWART T.S., DIEKMAN M.A., 1989. Effect of birth and fraternal litter zise and cross-fostering on growth and reproduction in swine. J. Anim. Sci., 67, 247-256.

\section{Summary}

Effect of fostering on survival, growth, reproduction and behavoural consequences in pigs.

Control of the mortality of surnumerary newborn pigs in modern intensive pig farming can be achieved by fostering. Its economical importance is high, concerning $5.6 \%$ of piglets born alive and 0.61 weaned piglet per litter.

This practice is increased by farrowing synchronisation and the necessity of improving the numerical productivity of pig farming.

Fostering has various consequences and particulary affects the behavior of transfered piglets. However, losses due to these behavioral disturbances are few. The main effect appears on growth performance on piglets until weaning and to a lesser extent until mar- ket. The unfavorable effects of this technique on reproductive performance are restricted to conception rate of gilts, to the total number of piglets born and to the number of piglets born alive.

Fostering leads to standardizing of litter size during lactation around an optimum which can vary according to the sow type. So, it seems to compensate the unfavorable effects of excessive litter size.

The period of transfer, the weight of transfered piglet and maternal care are the main factors which contribute to the success of fostering.

DEPRES E., CARITEZ J.C.,1991. L'adoption du porcelet, aspects comportementaux et zootechniques. INRA Prod. Anim., 4 (2), 177-182. 